\title{
Dynamique fluviale et dynamique écologique
}

\author{
J.R. Malavoi \\ Cabinet EPTEAU. Lyon \\ Y. Souchon \\ CEMAGREF BEA Laboratoire d'Hydroécologie Quantitative. Lyon
}

\section{E INTRODUCTION}

Il est aujourd'hui couramment admis que les processus physiques régissant la dynamique fluviale, et par conséquent la morphologie des cours d'eau et son évolution spatio-temporelle, régissent aussi, directement ou indirectement, la dynamique des écosystèmes qui leur sont associés.

Schématiquement, ces écosystèmes sont constitués :

— du lit mineur : partie du lit en eau pour des débits compris entre le débit d'étiage et le module

- du lit moyen ou bande active [1] : partie du lit en eau pour des débits compris entre le module et la crue de pleins bords et constituée de bancs alluviaux peu ou pas végétalisés, remaniés et rajeunis par des événement hydrologiques de fréquence moyenne à forte (entre la crue annuelle et la crue quinquennale). Cette bande active est souvent considérée comme une zone majeure de stockage temporaire de la charge alluviale de fond en transit.

— du lit majeur ou lit d'inondation : partie de la plaine alluviale mise en eau lors de crues de fréquence moyenne à rare.

Au sein de ce troisième type d'écosystème, il est parfois possible, pour des cours d'eau de rang supérieur, de distinguer des sous-systèmes, généralement constitués de formes relictuelles, témoins et indices de l'activité dynamique passée du cours d'eau, tels que les bras morts plus ou moins reliés au lit vif actuel, les marais (anciens bras morts comblés).

L'ensemble de ces écosystèmes, généralement interconnectés (par le réseau hydraulique de surface ou par le réseau souterrain) et dont l'origine, la structure et l'évolution sont intimement liées à la dynamique fluviale passée ou actuelle, peuvent être interprétés comme les éléments d'un système plus complexe : l'hydrosystème fluvial $[2,3]$. Les auteurs de ce concept, élaboré et testé sur le Rhône, indiquent qu'il est particulièrement adapté aux grands cours d'eau, ou tout au moins à ceux disposant d'une plaine alluviale suffisamment vaste pour permettre la création de la mosaïque d'écosystèmes cités plus haut, notamment les sous-systèmes du lit majeur.

L'objet de cet article est donc de montrer quels sont les liens extrêmement forts unissant, au sein de cet hydrosystème fluvial d'une grande complexité, la dynamique fluviale et la dynamique écologique. Il a aussi pour ambition de présenter, de manière non exhaustive, les risques induits par la modification des processus physiques et des variables de contrôle de la dynamique fluviale.

Le fonctionnement morpho-écologique de la portion de Loire comprise entre Villerest et Nevers illustrera cet exposé avant tout théorique.

\section{E ELEMENTS DE DYNAMIQUE FLUVIALE ET ECOLOGIQUE}

\subsection{Dynamique fluviale}

Dans des conditions naturelles, les rivières tendent à établir une combinaison stable entre leurs différentes variables constitutives [4] :

- les variables indépendantes ou variables de contrôle, - les variables dépendantes ou variables de réponse.

Parmi les variables de contrôle, deux sont fondamentales et régissent en grande partie la dynamique fluviale:

* le débit liquide : $Q$

* le débit solide : $\mathbf{Q}_{\mathbf{s}}$

La rivière cherche donc son équilibre entre le taux de charge alluviale imposé $\left(Q_{s}\right)$ et le débit liquide, énergie capable de l'évacuer $(Q)$ en ajustant sa géométrie en plan, en long et en travers.

D'autres variables de contrôle interviennent à divers degrés dans la «balance morphodynamique»:

* la pente globale de la vallée $\left(\mathrm{V}_{\mathrm{s}}\right)$, qui est une des composantes de l'énergie,

* les caractéristiques sédimentologiques du lit et des berges (D), qui conditionnent leur érodabilité au même titre que la végétation des berges $\left(B_{v}\right)$.

On suppose alors que tout cours d'eau dispose d'une gamme assez large de variables dites dépendantes, pour modeler sa morphologie en fonction des valeurs des paramètres indépendants et mettre en place une forme adéquate pour un transit optimal des débits liquides et de la charge solide entrant dans son système.

Ces variables dépendantes sont appelées aussi «degrés de liberté» d'ajustement aux variations des paramètres indépendants.

Hey [5] en distingue 7 : la largeur du lit (W), la profondeur moyenne (d), la pente du lit (S), la vitesse du courant $(\mathrm{V})$, la profondeur maximum $\left(\mathrm{d}_{\mathrm{m}}\right)$, la sinuosité (p), la lon- 


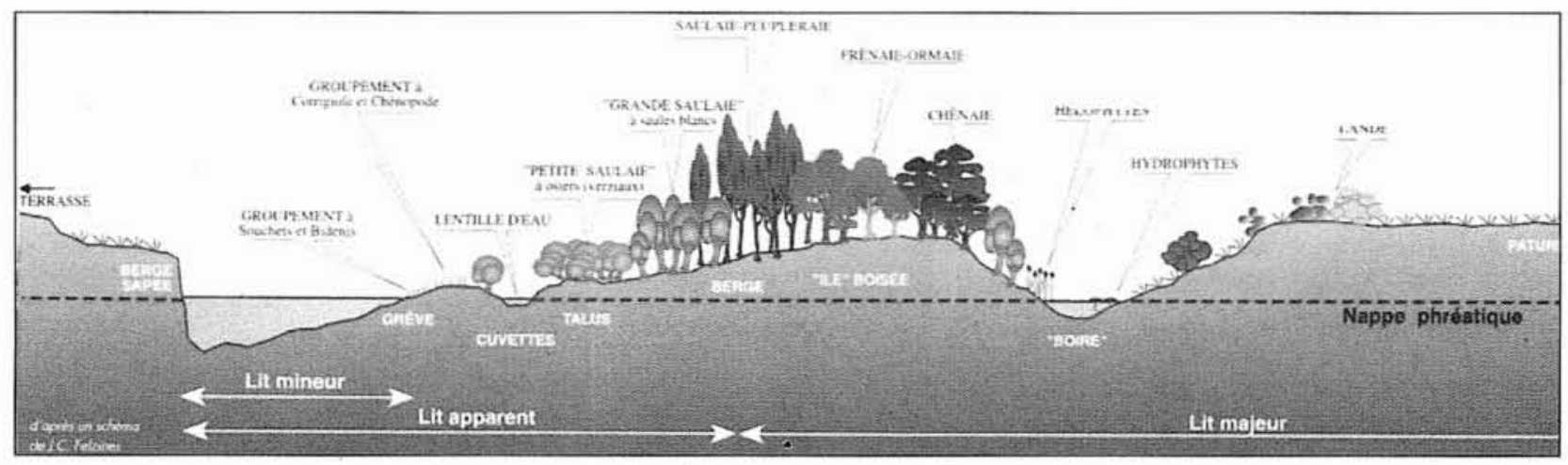

1. Coupe théorique du lit majeur de la Loire et indication de la répartition des séries végétales en fonction de la dynamique et de la topographie du lit [25]

gueur de l'arc du méandre ou l'espacement entre deux radiers $(\mathrm{z})$.

Par référence aux phénomènes observés en physique des systèmes ouverts, on dit alors que les rivières naturelles sont en «équilibre dynamique» ou en «quasi-équilibre» (selon l'echelle de temps choisie pour analyser ce phénomène) et qu'elles ajustent continuellement les valeurs de leurs variables dépendantes au gré des fluctuations naturelles (ou artificielles) de celles de leurs variables indépendantes.

Dans la réalité, $Q$ et $Q$ s ne sont pas les seuls paramètres impliqués dans la mise en action des processus d'ajustement ; ainsi toute modification de l'une des variables de réponse, ce qui est généralement le cas dans les aménagements de rivière ou dans les interventions humaines de type extraction, est en théorie susceptible d'entraîner par un processus complexe d'interactions et de rétroactions, une mutation de tout ou partie du système [6].

«Equilibre dynamique» ou «quasi-équilibre» signifie donc non pas absence totale de modification des caractéristiques physiques du cours d'eau mais au contraire ajustement permanent autour de conditions moyennes.

Ainsi sur un cours d'eau en équilibre dynamique ne doit-on pas s'étonner d'assister à des phénomènes d'érosion ou de dépôt, à des migrations vers l'aval des sinuosités, à l'exhaussement ou I'encaissement ponctuel du lit. Au contraire, tous ces phénomènes, ces pulsations pourrait-on dire, sont la preuve que le cours d'eau se porte plutôt bien et qu'il est bien toujours à la recherche de son équilibre.

$\mathrm{La}$ question fondamentale est donc d'identifier le seuil à partir duquel ces modifications géométriques ne sont plus liées au processus d'équilibre mais deviennent des indicateurs de dysfonctionnements du système.

La durée, voire même la possibilité de ces ajustements dynamiques, sont fonction notamment du type de cours d'eau touché (caractéristiques géologiques du bassin, régime hydrologique) et de sa puissance spécifique !

1 La puissance spécifique est calculée comme suit : $\omega=\gamma \mathrm{QJ} / 1$ en watts/m2 où $\gamma$ est le poids spécifique de l'eau $\left(\mathrm{N} / \mathrm{m}^{3}\right)$, $\mathrm{Q}$ le débit de pleins bords $\left(\mathrm{m}^{3} / \mathrm{s}\right)$, $J$ la pente du lit $(\mathrm{m} / \mathrm{m})$, I la largeur à pleins bords $(\mathrm{m})$. NB : on remplace parfois le débit de pleins bords par le débit de crue journalière de fréquence biennale qui lui est fortement corrélé...

\subsection{Dynamique écologique}

\subsubsection{Généralités}

Un hydrosystème fluvial naturel est caractérisé par une diversité géomorphologique dont le moteur est, notamment dans un cours d'eau à méandres, l'érosion des berges et la migration latérale du chenal vif [7]. Dans de nombreux cours d'eau, cette migration latérale s'effectue selon un axe amont-aval, l'ensemble du mouvement étant appelé translation $[8,9]$.

Dans ce processus de migration latérale ou aval, le chenal vif se déplace en érodant sa rive concave et en déposant des sédiments en transit sur sa rive convexe. On nomme ces zones de dépôt : bancs de convexité.

Quand les méandres atteignent un certain rayon de courbure, ils peuvent être recoupés naturellement à l'occasion de crues, même moyennes. Cette valeur seuil du rayon de courbure est variable selon le type de cours d'eau (fonction notamment de la pente, de la granulométrie du lit et des berges, de la végétation du lit majeur) mais la plupart des hydrosystèmes à méandres présentent ce type de processus. La partie de méandre ainsi recoupée devient alors un bras mort, dans un premier temps plus ou moins alimenté par le chenal vif, puis seulement par la nappe d'accompagnement ; il va progressivement se combler par apports de sédiments et de matière organique en période de crue, pour enfin faire partie intégrante du lit majeur sous la forme d'un marais ou d'une simple dépression topographique.

Ces processus d'érosion, transport de sédiments, dépôt, recoupement de méandre, ont pour effet de créer, détruire, recréer, à une échelle de temps comprise entre 10 et 100 ans en moyenne, une diversité de milieux dont la grande richesse écologique tient justement à leur fréquence de régénération et à leur assemblage sous forme de mosaïque sur une surface relativement restreinte (parfois seulement quelques milliers de $\mathrm{m}^{2}$ ).

En suivant la coupe transversale théorique du lit majeur d'un cours d'eau (figure 1), et sans entrer dans le détail des processus de colonisation des milieux et de successions écologiques, on observe généralement, en progressant du lit vif vers les marges externes du lit majeur : 
- le lit mineur presque toujours submergé et où ne se développe pas de végétation ligneuse et qui est considéré comme l'écosystème d'eau courante,

- le lit moyen, dont la submersion complète est généralement considérée comme étant de fréquence annuelle à quinquennale (en moyenne biennale). C'est le milieu où se succèdent spatialement et temporellement diverses espèces et strates végétales : les espèces herbacées pionnières, occupent les marges du lit actif et les bancs alluviaux fraîchement créés, elles sont relayées (dans l'espace et dans le temps) par les fourrés de saules et la ripisylve de saules blancs, régulièrement immergés, puis par la forêt d'aulnes.

- le lit majeur enfin, plus rarement inondé et où se développe la forêt alluviale des bois durs (frênes, ormes, chênes). Dans ce lit majeur, les sous-systèmes de bras morts, marais, dépressions topographiques, peuvent présenter une grande diversité d'associations végétales, depuis les roseaux jusqu'aux bois durs du stade ultime.

Une des grandes originalités écologiques des hydrosystèmes fluviaux, dont la Loire, se situe au niveau des lits mineurs et moyens, c'est-à-dire des parties de l'hydrosystème les plus fréquemment soumises aux processus d'érosion-transport-dépôt de la charge alluviale. Du fait du remaniement fréquent des sédiments déposés dans ces lits emboîtés, du fait de l'arrachage régulier des stades végétaux pionniers herbacés ou arbustifs, du fait de la grande variabilité saisonnière de la submersion de ces unités, on observe un rajeunissement régulier tant des formes fluviales que des successions végétales qui s'y développent et par conséquent des autres éléments des biocénoses qui y sont inféodés (oiseaux, insectes, mammifères).

Il faut noter enfin que ces milieux alluviaux mobiles sont parmi les seuls, avec les fronts de retraits des glaciers, les glissements de terrain et les coulées de lave, à offrir des possibilités de successions écologiques primaires ${ }^{2}$ [10].

La photographie aérienne présentée figure 2 illustre très clairement le rôle de la mobilité latérale du cours d'eau dans la création et le maintien de ces milieux à haute qualité écologique.

\subsubsection{Hydrodynamique et fonctionnement biologique}

\section{Des inondations bénéfiques pour l'hydrosystème}

C'est à l'occasion des crues de débordement que différents processus essentiels se réalisent : régénération du substrat, redistribution des accumulations de matière organique, dilution des particules fines minérales et organiques au sein de toute la plaine d'inondation. Ces épisodes sont mis à profit par les poissons pour accomplir certaines phases essentielles de leur cycle vital : migrations de montaison ou d'avalaison en fonction de la saison, reproduction dans les annexes fluviales, nutrition active dans un espace et avec une disponibilité trophique augmentés. Chez les poissons des grands cours d'eau, une des stratégies dominantes

2 successions débutant sur un substrat n'ayant encore jamais été colonisé, par opposition aux successions secondaires, prenant appui sur des substrats déja colonisés [10] consiste à se reproduire lors de la montée des eaux en gagnant les annexes fluviales, où les oeufs seront déposés. Les jeunes poissons s'y développeront jusqu'à être en mesure de gagner à leur tour le chenal plus vif. La durée de ces périodes de hautes eaux conditionne le succès du recrutement annuel (nombre de jeunes qui vont fonder une cohorte). Une relation très nette entre nombre de jours d'inondation et biomasse de poissons a été établie pour le Danube par Stankovic et Jankovic [11]. Ce type de résultat, également mentionné par Léger [12] pour la Saône prend de plus en plus une connotation historique, au vu de la raréfaction des plaines alluviales européennes encore fonctionnelles.

Des épisodes hydrologiques extrêmes tolérés par la faune grâce à la diversité et à la complémentarité des formes fluviales

Lors d'étiages ou de crues exceptionnels, certaines unités morphodynamiques du lit mineur comme les profonds (étiages) ou du lit majeur comme les annexes fluviales (crues) servent de refuges au peuplement de poissons. Elles contribuent ainsi à la perrénité des espèces. Les invertébrés benthiques rhéophiles, organismes de plus petite taille adulte, cherchent quant à eux refuge dans le substrat, à condition qu'il soit suffisamment ouvert et non colmaté en profondeur. Cette bonne qualité de substrat est également bénéfique pour certaines espèces de poissons qui enfouissent leurs oeufs dans les graviers. Ces conditions d'habitabilité du substrat sont garanties par une dynamique fluviale active qui participe à un renouvellement des formes et à une régénération des dépôts sédimentaires.

La diversité des habitats, garante de la diversité biologique

Cette relation a été démontrée pour les cours d'eau par Gorman et Karr [13]. Une plus grande diversité de combinaisons des hauteurs d'eau, vitesses du courant, paramètres de l'écoulement et des faciès, paramètres de la morphologie s'accompagne d'une plus grande diversité spécifique et fonctionnelle chez les poissons. Davantage de stratégies vitales, de modes d'utilisation de l'espace et des ressources peuvent cohabiter dans ces environnements physiques.

C'est cette diversité de milieux, leur rajeunissement permanent, leur évolution spatio-temporelle si particulière, qu'il convient de préserver en garantissant au maximum les fonctionnalités morphogéniques des cours d'eau.

\section{I DYNAMIQUE FLUVIALE ET ECOLOGIQUE DE LA LOIRE ENTRE VILLEREST ET LE BEC D'ALLIER}

A titre d'exemple concret des divers aspects théoriques présentés plus haut, nous avons choisi d'exposer l'état de nos connaissances concernant la LOIRE dans la partie de son cours située entre Villerest et Nevers.

Le fonctionnement de la LOIRE dans ce secteur d'étude revêt un caractère exceptionnel car c'est à notre connaissance le seul fleuve de cette taille en France, présentant, sur une aussi grande longueur (environ $200 \mathrm{~km}$ ) et dans 

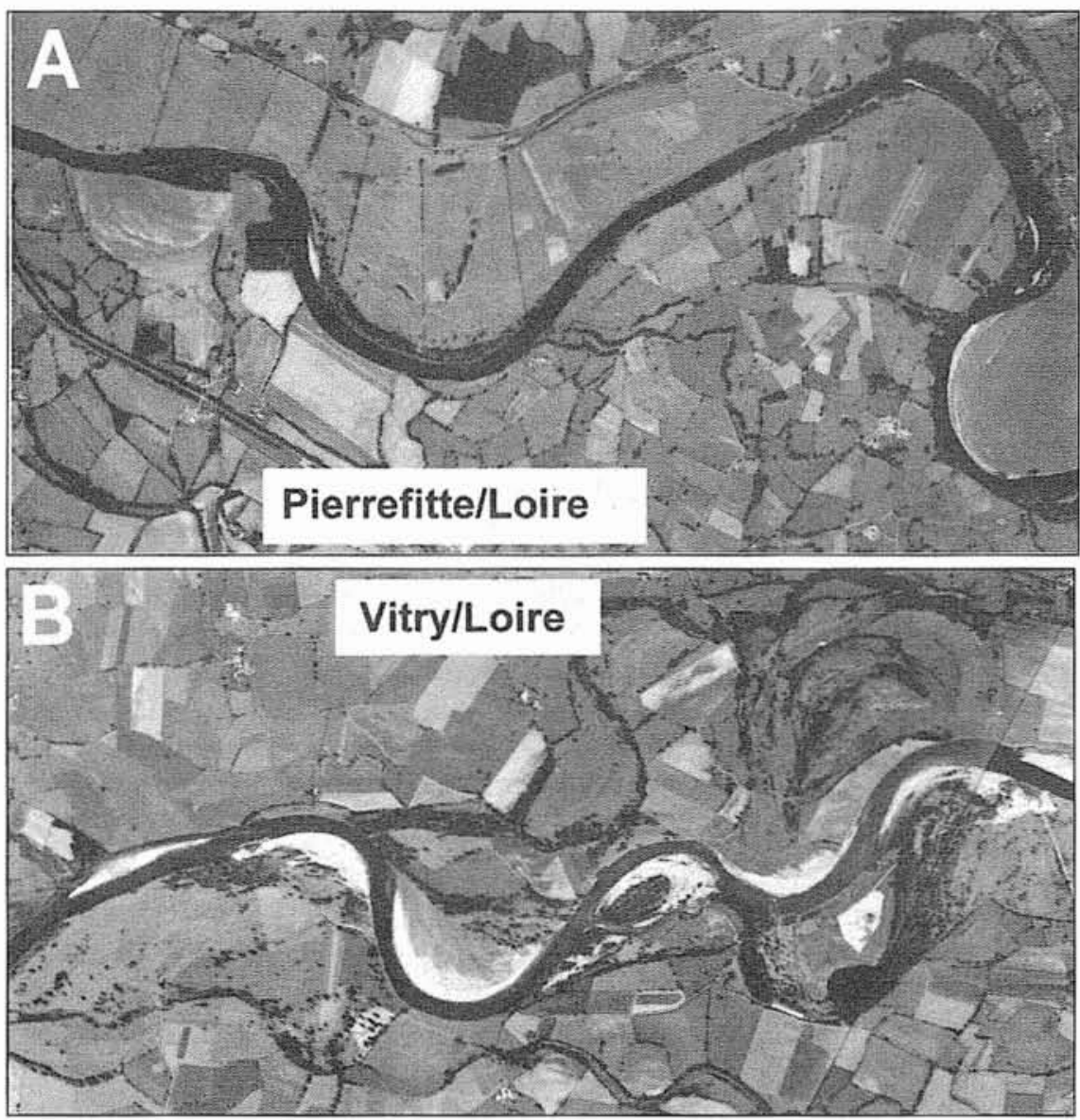

$1 \mathrm{~km}$

une gamme de pentes aussi faibles (environ $0.5 \mathrm{~m}$ par kilomètre), un style fluvial globalement méandriforme et généralement à forte activité dynamique.

Le linéaire de Loire compris dans la zone d'étude avoisine donc les 200 kilomètres. Ce linéaire est trop important et présente une trop grande variété de physionomies et de fonctionnements morphodynamiques pour être analysé immédiatement dans son ensemble. Il est donc indispensable, tant pour le diagnostic que pour la définition d'une logique d'aménagement, d'effectuer une sectorisation de la Loire en unités présentant une certaine homogénéité sur le plan des variables morphologiques et hydrologiques.

Cette sectorisation en «tronçons», nous permet de raisonner par secteurs homogènes de taille réduite (quelques dizaines de kilomètres), tout en conservant la vue d'ensemble du cours d'eau et de son continuum amont-aval.

Six «tronçons» ont été délimités sur des critères géologiques, géomorphologiques et hydrologiques. Cette sectorisation globale recoupe fréquemment celle proposée par Maire et al. [14] et SOGREAH [15] dans le cadre de leur étude de la Loire en Bourgogne.

Ces tronçons ont eux-mêmes été subdivisés en «sous-tronçons» sur la base de l'intensité de la dynamique fluviale.

Trois niveaux d'intensité ont été distingués :
2. Vue en plan de deux portions de Loire distantes d'une trentaine de kilomètres. La première (A) est incluse dans un sous-tronçon à dynamique latérale actuellement inexistante : on constate l'absence de variété des formes fluviales (pas de bancs de convexité).

La seconde (B) se situe dans un sous-tronçon à dynamique active : on observe une mosaïque de milieux garante d'une grande diversité écologique (bancs alluviaux vifs ou à végétation pionnière, saulaie basse, forêt à bois durs etc.)
- Niveau I (codé N I dans le texte) : sous-tronçon à dynamique faible ou inexistante. Sur l'ensemble du soustronçon, le lit est globalement, naturellement ou artificiellement, contraint latéralement et/ou verticalement. Il n'existe pas d'indices d'activité dynamique récente. Il n'existe que peu ou pas de zones d'accumulation alluviale (bancs vifs). Le tracé du lit de la Loire correspond généralement aux limites administratives (département, canton, commune), ce qui confirme sa grande stabilité spatio-temporelle (stabilité sur au moins 200 ans).

Ce type de dynamique stabilisée représente actuellement $50 \%$ du linéaire du secteur d'étude.

- Niveau II (codé N II) : sous-tronçon à dynamique modérée. Le lit est potentiellement actif mais il existe des contraintes locales (naturelles ou artificielles) qui grèvent en partie les possibilités d'expression de cette dynamique. On y observe cependant des processus d'érosion/dépôt.

Ce type de dynamique en voie de stabilisation représente actuellement $15 \%$ du linéaire du secteur d'étude.

- Niveau III (codé N III) : sous-tronçon à dynamique active. Le lit est globalement actif sur l'ensemble du soustronçon mais il existe quelques contraintes locales (naturelles ou artificielles) qui peuvent entraver localement les processus d'érosion. 
Les sous-tronçons à dynamique globalement active représentent $35 \%$ du linéaire de la Loire entre Villerest et le Bec d'Allier.

La figure 3 présente la sectorisation actuellement proposée [16]. Nous y avons aussi reporté les limites des portions de cours d'eau à haute qualité écologique, à partir des cartes de synthèse de l'étude menée par le GREMINAT entre 1981 et 1983 [17] ${ }^{3}$.

\section{- 3.1 Tronçon I : de Villerest à Pouilly S/Charlieu : $23.8 \mathrm{~km}$. N I}

Ce premier tronçon commence au pied du barrage de Villerest et s'achève à l'amont immédiat de la confluence avec le Sornin. Le barrage marque la brusque transition entre le Massif Central cristallin et le bassin d'effondrement de la Loire (Limagne) dont le remplissage, comme celui de la Limagne d'Allier, est constitué d'accumulations tertiaires et quaternaires. Long de 24 kilomètres environ, le lit mineur y présente une pente de l'ordre de 0.001 ce qui en fait le tronçon le plus pentu de toute la zone d'étude. Malgré un fond de vallée relativement large en aval de Roanne $(2-2.5 \mathrm{~km})$, ce tronçon est globalement stable en plan (quelques érosions latérales) mais est marqué par une incision liée aux exploitations de graviers des décennies précédentes $(-1 \mathrm{~m}$ au minimum à Roanne). Il n'a pas été subdivisé en sous-tronçons en raison de la relative homogénéité du fonctionnement morphodynamique.

Le lit est actuellement stable en plan malgré quelques érosions de berges localisées. Certains indices témoignent cependant d'une activité latérale passée assez forte qui semble s'être stabilisée depuis (la limite cantonale au droit du chateau de Vougy indique la présence d'un ancien méandre de grande amplitude qui n'était déjà plus actif en 1850).

D'un point de vue écologique global [17] ce tronçon ne présente pas d'intérêt majeur.

\subsection{Tronçon II : de Pouilly à Iguerande : 8.1 km}

Ce second tronçon est caractérisé par l'encaissement du cours de la Loire dans le «défilé d'Iguerande» (traversée de formations du jurassique moyen). Il s'achève au droit du lieu dit «la Rivolière». La largeur du fond de vallée est de l'ordre de 600-800 m ce qui n'autorise qu'une divagation de faible amplitude. La pente se réduit de moitié pour passer à environ 0.0005 .

Comme dans le précédent tronçon, la dynamique fluviale semble peu active en plan, malgré quelques érosions de

\footnotetext{
${ }^{3}$ Au terme d'une étude quasi-exhaustive de la faune et de la flore de la vallée de la Loire entre Iguerande et Cosne, les auteurs concluaient à une richesse biologique considérable de l'ensemble de la vallée, malgré une évolution récente des paysages (uniformisation liée à l'évolution agricole vers le système céréalier) et un appauvrissement probable de cette richesse en raison des diverses interventions humaines inventoriées à l'époque (extractions. aujourd'hui arrêtées, barrages, urbanisation des lits majeurs, protection des berges, motonautisme, "moto verte» sur les bancs et dans la forêt alluviale). Ils indiquaient cependant un certain nombre de secteurs, plus ou moins étendus, caractérisés par une grande richesse et originalité floristique et un grand intérêt pour la faune terrestre, notamment l'avifaune.
}

berges localisées et de faible amplitude. Ce tronçon présente une telle homogénéité sur le plan du fonctionnement morphodynamique et écologique qu'il n'a pas été utile de le subdiviser.

L'activité fluviale y est actuellement modeste et ce tronçon ne présente qu'une qualité écologique globale moyenne.

\subsection{Tronçon III : d'Iguerande à Digoin : 42.6 km}

Ce tronçon marque l'entrée dans le «val libre» de Loire. Il s'achève à Digoin, en amont immédiat de la confluence avec l'Arroux et présente une pente moyenne de 0.0006 . La largeur du fond de vallée est de l'ordre de 2-3 kilomètres, cette valeur correspondant à l'espace de divagation théorique du lit actif. Ce tronçon, globalement caractérisé par une dynamique fluviale active, a néanmoins été subdivisé en 6 sous-tronçons présentant des différences importantes au niveau de l'activité fluviale actuelle.

Cette sous-sectorisation, basée sur des critères morphodynamiques, correspond presque exactement à celle proposée par Bugnon et al. [17] pour définir les zones à haute qualité écologique globale (intérêt pour l'avifaune et richesse floristique).

\subsubsection{Sous-tronçon III.1 $(4.8 \mathrm{~km}):$ N III}

De 'la Rivolière' à Artaix ('le Port'), ce premier soustronçon sera plutôt qualifié d'actif, même si les taux d'érosion et de migration enregistrés actuellement sont assez faibles. On y observe cependant de vastes bancs alluviaux vifs.

La qualité écologique globale est très forte.

\subsubsection{Sous-tronçon III. $2(8.3 \mathrm{~km}): \mathrm{N} \mathrm{I}$}

D'Artaix 'le Port' à Bourg le Comte $(250 \mathrm{~m}$ en amont de la confluence avec l'Urbise), ce sous-tronçon présente une grande stabilité en plan et relativement peu de bancs alluviaux, forêts ou même simple ripisylve.

La qualité écologique globale est moyenne.

\subsubsection{Sous-tronçon III.3 $(2.7 \mathrm{~km})$ : N III}

Du point précédent à Avrilly ('les Bouillets') ce petit sous-tronçon est intéressant dans la mesure où il s'agit d'un secteur à dynamique active inclus dans un plus vaste linéaire globalement peu dynamique.

La qualité écologique redevient ponctuellement bonne.

\subsubsection{Sous-tronçon III.4 $(3.5 \mathrm{~km}): \mathrm{N} \mathrm{I}$}

De faible développement, ce sous-tronçon, du point précédent au pont de Bonnand, est stable car soit protégé, soit calé contre le versant rocheux en rive gauche. La concordance entre les limites administratives et le tracé du lit actuel indique que cette stabilité est acquise depuis longtemps.

La qualité écologique est moyenne. 


\section{Sectorisation morphologique de la LOIRE entre Roanne et Nevers}

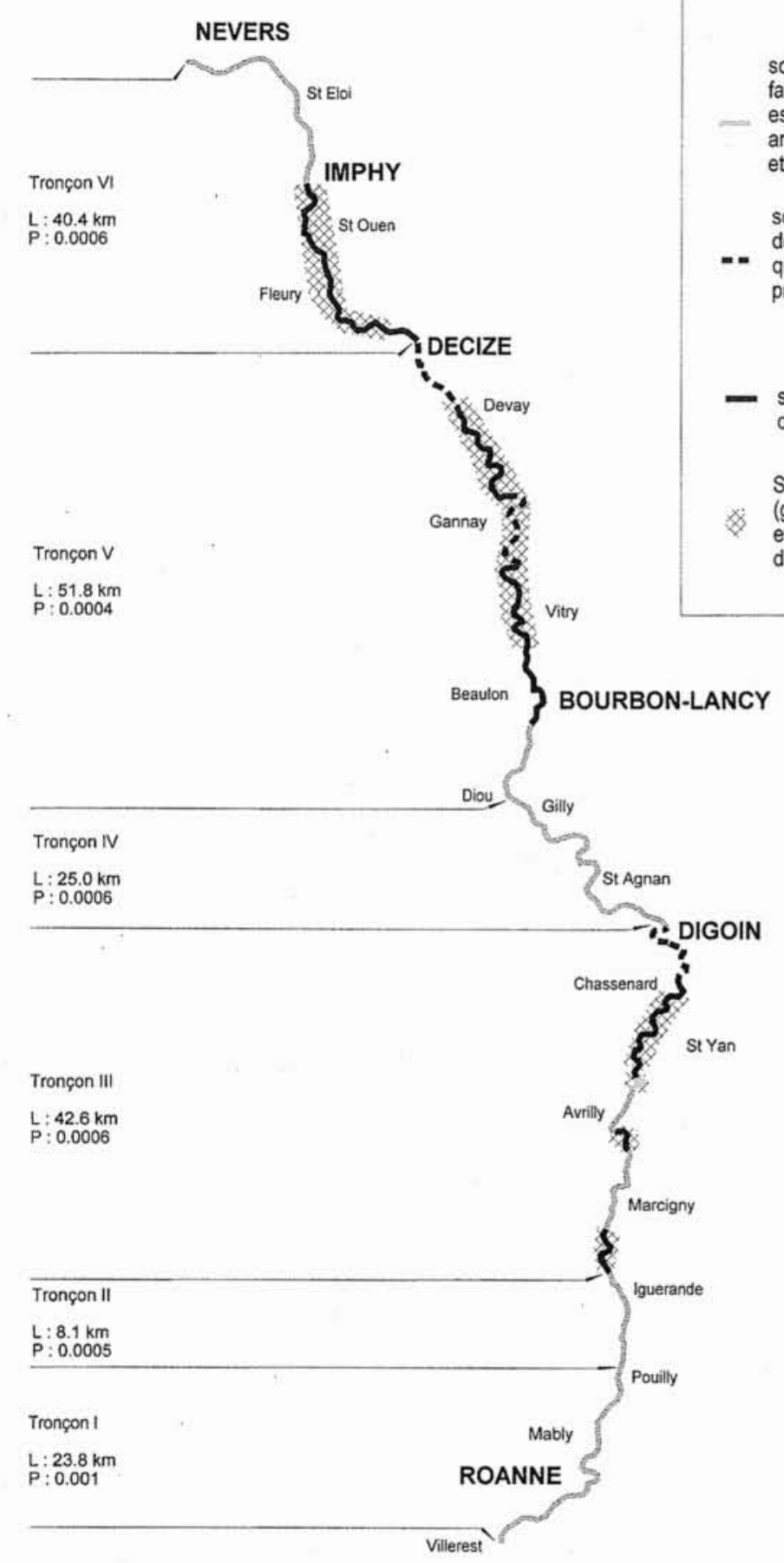

Limites de tronçons

sous-tronçon à dynamique

faible ou inexistante (le lit mineur

est majoritairement, naturellement ou

artificiellement, contraint latéralement

et/ou verticalement)

sous-tronçon à dynamique modérée (il existe

des contraintes latérales et/ou verticales,

- qui entravent en partie le développement des processus d'érosion)

sous-tronçon à dynamique active (malgré l'existence de contraintes ponctuelles)

Secteur à haute qualité écologique globale

(grand intérêt pour l'avifaune, richesse

et originalité floristique élevée)

d'après GREMINAT (1983)

3. Sectorisation de la LOIRE en tronçons et sous-tronçons entre Roannes et Nevers. 


\subsubsection{Sous-tronçon III.5 $(13.2 \mathrm{~km}): \mathrm{N}$ III}

Du pont de Bonnand à Varenne St Germain (confluence avec l'Arconce, ce grand sous-tronçon présente une dynamique fluviale assez intense sur le long terme, bien qu'il n'ait que peu évolué ces dernières années. Certains méandres sont actuellement protégés (épis en aval immédiat du Pont de Bonnand) ou calés contre le substratum rocheux (les 'Gonons' à Chassenard). Les bancs alluviaux sont cependant nombreux et vastes.

La qualité écologique globale est très bonne, particulièrement pour ce qui concerne l'avifaune. Subsistent cependant les stigmates d'extractions en lit mineur ou moyen qui grèvent encore la qualité de cet ensemble.

\subsubsection{Sous-tronçon III.6 $(10.1 \mathrm{~km})$ : N II}

De la confluence de l'Arconce à celle de l'Arroux, ce dernier sous-tronçon marque l'entrée dans la zone proximale de Digoin. La dynamique naturelle devrait être ici un méandrage actif avec translation et recoupement de méandres. Des aménagements récents viennent modifier les processus naturels (enrochement de deux méandres de grande amplitude en amont immédiat de Digoin). Sauf en 2 points, qui seront d'ailleurs à traiter (méandre de Chassenard et méandre de Molinet), la dynamique latérale semble actuellement très compromise.

De ce fait, ce sous-tronçon ne présente pas, ou plus, sa grande qualité écologique potentielle.

\subsection{Tronçon IV : de Digoin à Diou : 25 km}

Ce tronçon est marqué par l'infléchissement vers le NordOuest de la vallée de la Loire, infléchissement dû au contournement par l'ouest du horst cristallin du Morvan. Le tronçon s'achève à Diou, au niveau du seuil rocheux affleurant en fond du lit actif et calant très fortement le plancher alluvial, en amont immédiat de la confluence avec la Besbre. Le fond de vallée présente une largeur de l'ordre de 2-3 kilomètres, l'espace de divagation théorique étant fortement contraint, au delà de cette largeur, par ses deux coteaux latéraux. La pente reste du même ordre que précédemment : 0.0006 .

La caractéristique majeure de ce tronçon est d'être extrêmement stable en plan puisque le lit actif n'a quasiment pas évolué depuis la carte de 1850 . Il s'agit probablement du secteur le plus stable de toute la zone d'étude. Cette stabilité s'explique tant par des contraintes naturelles (affleurements en pied de coteaux, seuil rocheux) que par des aménagements anciens stabilisant les méandres (perrés, enrochements). De même que les tronçons I et II, sa grande homogénéité de comportement morphodynamique ne justifie pas de le subdiviser.

Comme toutes les unités à dynamique fluviale peu active, ce tronçon ne présente pas une grande qualité écologique.

\subsection{Tronçon V : de Diou à Decize : 51.8 km}

Du seuil de Diou à la confluence avec l'Aron, ce tronçon est celui présentant la dynamique fluviale la plus active sur le linéaire le plus important. Les méandres sont presque tous actifs (érosion latérale intense à très intense) dans un fond de vallée avoisinant les 4 à 5 kilomètres de large. L'intensité de sa dynamique fluviale est reconnue et analysée depuis longtemps $[18,14]$. Son exceptionnelle qualité écologique globale est aussi fermement établie (Loire Nature, 1995). Compte tenu du fort développement des méandres, la pente du lit se réduit assez fortement pour passer à des valeurs de l'ordre de 0.0004 .

Ce tronçon a été subdivisé en 5 sous-tronçons, certains secteurs présentant des contraintes latérales limitant les possibilités d'évolution en plan.

\subsubsection{Sous-tronçon V.1 $(7.1 \mathrm{~km}) \mathrm{N} \mathrm{I}$}

Ce sous-tronçon correspond à une zone de transition entre le tronçon précédent à dynamique faible le long des contreforts du Morvan et la plaine bourbonnaise dont la dynamique fluviale active est reconnue depuis fort longtemps [14]. Du seuil de Diou (amont de la confluence avec la Besbre) à Beaulon ('Valatte') ce sous-tronçon présente encore les caractéristiques de stabilité du tronçon IV mais les nombreux anciens tracés observables dans le lit majeur actuel en rive gauche indiquent une intense activité fluviale à une époque reculée (le tracé de 1850 était déjà dans sa position actuelle).

La qualité écologique de ce premier sous-tronçon est qualifiée de moyenne par Bugnon et al. [17].

\subsubsection{Sous-tronçon V.2 (17.3 km) N III}

Ce sous-tronçon s'étend de Beaulon ('Valatte') à Cronat ('Ballore'). Il s'agit là d'un des sous-tronçons les plus actifs, et sur la plus longue distance, du secteur d'étude. La dynamique fluviale est caractérisée par une évolution rapide des méandres avec des taux d'érosion avoisinant les 50 à $90 \mathrm{~m}$ en 10 ans.

C'est ce sous-tronçon qui a connu la modification géomorphologique la plus importante de ces 50 dernières années avec le recoupement de la série de méandres des 'Grands Verziaux' en 1957. Ce recoupement spectaculaire a modifié un paramètre morphodynamique majeur, la pente du lit (celle-ci a été multipliée par 2), ce qui a entraîné une érosion régressive et progressive dont on ne connaît encore pas précisément l'amplitude mais qui est probablement stoppée en amont par le seuil de Diou.

Malgré cette activité dynamique intense, la première partie de ce sous-tronçon (jusqu'à Lesme) ne semble pas présenter une qualité écologique majeure sauf très ponctuellement (dans l'ancien méandre des grands Verziaux et localement sur quelques bancs de convexité).

La seconde partie du sous-tronçon présente dans son ensemble une haute qualité écologique globale.

\subsubsection{Sous-tronçon V.3 $(8.2 \mathrm{~km}) \mathrm{N} \mathrm{II}$}

De Cronat ('Ballore') à St Hilaire Fontaine ('Thareau'), la mobilité latérale de la Loire est régulièrement contrainte par 
des digues ('Ballore', 'Thareau') ou des protections de berges (le long de la D15 ('le grand Gannay') ainsi qu'à 'Champfort' sur la commune de Gannay). Ce sous-tronçon est donc globalement classé en classe d'intensité N II. Il existe cependant quelques zones d'érosion latérale et les bancs alluviaux restent très fréquents, ce qui explique que sa qualité écologique soit très bonne malgré ces contraintes locales.

\subsubsection{Sous-tronçon V.4 $(10 \mathrm{~km}) \mathrm{N} \mathrm{III}$}

De St Hilaire Fontaine ('Thareau') à Decize ('Domaine Durand', limite de la commune de Cossaye), le lit redevient très actif et présente de nombreux méandres alternés et de fréquentes zones d'accumulation (bancs vifs). La forêt alluviale offre là un de ses plus grands développements longitudinaux continus, ce qui fait probablement de ce sous-tronçon l'un des plus intéressants d'un point de vue écologique de l'ensemble de la zone d'étude.

\subsubsection{Sous-tronçon V.5 $(10 \mathrm{~km}) \mathrm{N}$ II}

Du 'Domaine Durand' à Decize (confluence de l'Aron), c'est le dernier sous-tronçon du vaste tronçon $\mathrm{V}$. La dynamique du lit n'est plus aussi active que précédemment, pour différentes raisons déjà développées dans l'étude morphodynamique de Maire et al. [14] : contraintes structurales liées à la traversée du horts de Decize (affleurements rocheux), réduction de la capacité érosive du fleuve par le calage de la ligne d'eau lié au barrage de Decize, aménagements lourds dans la traversée de l'agglomération (digues en rive gauche et droite).

Malgré cela, quelques érosions localisées peuvent être observées et de nombreux bancs alluviaux vifs se développent sur l'ensemble du sous-tronçon.

La qualité écologique globale est moyenne sauf localement (amont de Brain, île de 'Chevannes') ou elle est très bonne en termes d'originalité et de richesse floristique.

\section{ค 3.6 Tronçon VI : de Decize au Bec d'ALLIER : 40,4 km}

Dernier tronçon de la zone d'étude, il marque la transition avec le «val endigué» de Loire (par opposition au «val libre» de l'amont), au delà de Nevers, dont le style morphologique est notablement différent (tressage). La pente redevient assez forte : 0.0006 . Il a été subdivisé en deux soustronçons.

\subsubsection{Sous-tronçon VI.1 (19.6 km) N III}

De la confluence de l'ARON à Imphy ('le Grand Vernay') la Loire présente une succession de sinuosités de grande longueur d'onde mais de faible amplitude. Certaines sont actuellement très actives mais d'autres sont stabilisées par des enrochements ('Tinte', sur la commune de Sougy, 'Mussy' sur la commune d'Avril s/Loire). La partie terminale du sous-tronçon est calée en rive gauche par la digue
d'Uxeloup contre laquelle la Loire butte actuellement ('Domaine de la Grève').

Malgré ces quelques contraintes, ce sous-tronçon est globalement actif et est classé comme de grand intérêt écologique.

\subsubsection{Sous-tronçon VI.1 $(20.4 \mathrm{~km}) \mathrm{N} \mathrm{I}$}

D'Imphy ('le Grand Vernay') au Bec d'Allier, la Loire ne présente plus de signes d'activité latérale et ceci depuis fort longtemps comme en témoignent les limites administratives (communales et cantonales) calées sur le tracé du lit mineur actuel. L'étude morphodynamique de 1983 diagnostiquait un enfoncement du lit lié à d'anciennes extractions et à des affleurements rocheux en rive droite, et qui expliquerait cette grande stabilité en plan.

La qualité écologique de l'ensemble du sous-tronçon est considérée comme moyenne, sauf très ponctuellement ('Harlot').

\subsection{Peuplement de poissons dans cette portion de Loire}

La connaissance des peuplements de poissons du fleuve Loire est trop parcellaire pour permettre une analyse conjointe avec sa morphologie. Cette absence de recensement ne doit toutefois pas constituer un alibi pour ignorer la richesse potentielle de certains des secteurs identifiés, que l'on peut soupçonner en adoptant un raisonnement analogique avec des systèmes fluviaux mieux décrits (Rhône, Danube, voire Rhin).

Lors de sondages par pêche électrique en 1980 et 1981, Souchon et al. [19] avaient caractérisé le peuplement de poissons en deux stations incluses dans le secteur étudié. Il s'agissait de l'amont de Digoin (Chassenard, sous-tronçon III.5) et de St Aubin à l'aval de Decize (sous-tronçon VI.1). Le peuplement global comprenait 27 espèces de téléostéens et 2 cyclostomes, la Lamproie de Planer et la Lamproie marine (aval Decize). Il était majoritairement constitué de cyprinidés rhéophiles comme le chevaine, le barbeau, le hotu et la vandoise. Parmi les espèces remarquables du secteur, on relève encore un grand migrateur, la grande Alose qui se reproduit à l'aval de Decize, faute de pouvoir franchir le barrage de Decize-St Léger les Vignes [20]. Elle dépose ses oeufs dans le chenal principal dans des graviers $(2 \mathrm{~mm}$ à $2 \mathrm{~cm})$ de mai à juillet à une température supérieure à $12{ }^{\circ} \mathrm{C}$. Deux espèces introduites de la famille des centrarchidés sont aussi présentes : le Black-bass (Decize), inféodé à l'existence de bras latéraux et le Crapet des roches (Ambloplites rupestris) à Digoin, dont c'est l'une des seules stations recensées en France [21]. Les tendances récentes d'évolution qualitative du peuplement sont données par Roché et Heintz [22] : amélioration potentielle du franchissement des aloses par aménagement d'une passe à poisson au barrage sus-cité à partir de 1993, d'où l'importance de maintenir dans le fleuve à l'amont des grandes plages de gravier se régénérant ; bon maintien des espèces rhéophiles telles que le goujon, l'ablette, le spirlin et la vandoise ; certaine régression du sandre et augmentation de la 
brème bordelière ; expansion du silure depuis 1990, enfin difficultés pour le brochet très dépendant pour sa reproduction de l'inondation des prairies au printemps (enfoncement du lit ou moindre accessibilité aux herbiers des bras secondaires ?). Un suivi plus quantitatif inscrit dans la durée et réparti en fonction de la sectorisation morphologique renforcerait avec avantage les critères de repérage et de sélection des zones fluviales remarquables.

\section{CONCLUSION : UN CONCEPT DE GESTION : L'ESPACE DE LIBERTE}

Afin de préserver le fonctionnement morphodynamique des cours d'eau et les richesses biologiques qui lui sont intimement liées, il convient de proposer une gestion des plaines alluviales basée sur le concept d'espace de liberté.

Ce concept avait été fermement adopté comme principe de gestion à l'occasion des Assises Nationales de l'Eau [23]. Il fait aussi partie des préconisations du SDAGE du bassin LOIRE-BRETAGNE.

\subsection{Définition}

Tout cours d'eau naturel de type méandriforme ou sinueux (hormis ceux naturellement contraints : gorges) dispose sur les marges de son lit actif, d'une zone d'expansion qui lui permet de maintenir un tracé en plan en équilibre par rapport aux débits liquides et solides qui lui sont imposés par la nature et qui sont les deux variables essentielles de contrôle de la dynamique et de la morphologie fluviales (cf partie théorique). Cet espace est aussi l'une des sources principales d'alimentation des cours d'eau en charge solide (notion de substitution de charge par érosion des berges et du fond).

Deux concepts, non exclusifs l'un de l'autre, permettent de définir cet espace d'expansion ;

* un concept géomorphologique : l'espace de divagation maximale théorique du cours d'eau [24] : il s'agit de l'ensemble du fond de vallée qui par sa nature géologique (alluvions récentes ou anciennes, roches érodables) peut être soumis à l'activité érosive de la rivière et être le lieu d'ajustements en plan du tracé. Cet espace peut être très vaste et ne pas être entièrement utilisé par la rivière à notre échelle de temps,

* un concept de gestion : l'espace de liberté [23] : ce concept est dérivé du précédent. L'espace de liberté est de largeur inférieure ou égale à celle de l'espace de divagation théorique maximale du cours d'eau. Il s'agit de l'espace minimal à préserver pour permettre au cours d'eau de conserver son potentiel d'ajustement en plan et en long en fonction de l'évolution des autres variables ( $Q$ et $Q_{s}$ ).

La largeur minimale de cet espace de liberté peut être évaluée à 10 à 15 fois la largeur du lit actif à pleins bords. Cette valeur correspond à l'amplitude moyenne de nombreux trains de méandres ayant pu être analysés par un grand nombre de géomorphologues (la littérature scientifique anglo-saxonne en est particulièrement prodigue ).

Si cet espace de liberté minimal n'est pas respecté (endiguement étroit, enrochements de deux berges opposées), on peut s'attendre à de nombreuses modifications morphologiques du cours d'eau telles que l'enfoncement du lit par érosion progressive ou régressive, liée à l'augmentation des forces tractrices en crue (non débordement biennal), à la réduction de la charge alluviale de substitution, à l'augmentation de la pente.

Il est admis aujourd'hui que la plupart des cours d'eau «vivent» essentiellement sur le stock alluvionnaire disponible dans les fonds de vallée (alluvions récentes et basses terrasses pour la Loire) qui leur permet, par le processus d'érosion des berges, de conserver un fonctionnement relativement équilibré et de freiner le processus d'enfoncement généralisé du lit. Il est donc clair que la réduction de l'espace de liberté (endiguement, enrochements), et donc la limitation du processus d'érosion latérale, ne pourra qu'accélérer le phénomène d'enfoncement inéluctablement lié au déficit de charge actuel.

\subsection{Impacts liés à la réduction de l'espace de liberté}

Les modifications morphologiques induites par la réduction de l'espace de liberté outre l'impact majeur qu'elles peuvent avoir sur l'hydrologie (augmentation des pointes de crues du fait de la réduction des zones naturelles d'écrêtement en cas de présence de digues) sur l'hydraulique (modification du régime du transport solide), sur les ouvrages d'arts (effondrement de ponts, de digues, de seuils de moulin etc..) ont aussi des impacts écologiques majeurs qu'il convient de ne pas négliger.

Ces impacts écologiques sont multiples, nous n'en développerons donc que trois à titre d'exemple :

- l'enfoncement du lit signifie enfoncement de la nappe d'accompagnement, dépérissement de la forêt alluviale à bois tendres et remplacement par un cortège d'espèces à bois durs, donc à une banalisation des milieux puisque ces espèces sont déjà présentes en dehors de la plaine alluviale [1].

- la réduction de l'espace de liberté du cours d'eau a pour effet de stopper de manière quasi irréversible (réversibilité fonction de la nature de l'aménagement) le processus de rajeunissement permanent des formes alluviales et de leur végétation associée (jeunes stades de saules, aulnes, peupliers). Ce rajeunissement lié aux crues et à la migration du chenal vif est le garant d'une diversité maximale des milieux et donc de la faune aquatique et terrestre qui leur sont associées. La constriction de l'espace de liberté signifie donc à court terme le dépérissement et la disparition de toute la faune et la flore inféodées aux milieux alluviaux mobiles.

- cela entraîne aussi un abandon accéléré des bras morts ou latéraux dont le rôle écologique est fondamental (reproduction de certaines espèces de poissons, zones de refuge en période de crue). 


\section{Bibliographie}

[1] BRAVARD J.P. - La basse vallée de l'Ain : Dynamique fluviale appliquée à l'écologie. In Doc. Cartogr. Ecol. 29, 17-43 (1986).

[2] ROUX A.L. (ed) - Cartographie polythématique appliquée à la gestion écologique des eaux: étude d'un hydrosystème fluvial : le Haut-Rhône français. Edit. CNRS, Centre Région. Publ. Lyon, 116 p. (1982).

[3] AMOROS C., ROUX A.L., REYGROBELLET J.L., BRAVARD J.P., PAUTOU G. - A method for applied ecological studies of fluvial hydrosystems. Regulated Rivers 1, 17-36 (1987).

[4] SCHUMM S.A. - The shape of alluvial channels in relation to sediment type. US Géol. Surv. Prof. Paper. 352 B, 16-30, (1960).

[5] HEY R.D. - River mechanics. JIWES 40, 2, 139-158.(1986)

[6] BRAVARD J.P., PETTS G.E. - Interférences avec les interventions humaines. In Hydrosystèmes fluviaux. Sous la direction de C. Amoros et G.E. Petts. Masson ed.. Paris. 233-253 (1993).

[7] BRAVARD J.P., GILVEAR D.J. - Structure hydro-géomorphologique des hydrosystèmes. In Hydrosystèmes fluviaux. Sous la direction de C. Amoros et G.E. Petts. Masson ed.. Paris. 83-103 (1993).

[8] HOOKE J.M., HARVEY A.M. - Meander changes in relation to bend morphology and secondary flows. In Modern an ancient fluvial systems, COLLINSON J.D., LEWIN J., ed., Blackwell Scientific Publications, Cambridge, 121-132. (1983).

[9] MALAVOI J.R. - La basse vallée de l'Ain : Etude hydrologique et morphodynamique. Mém. Maîtrise. Université Lyon III. (1985).

[10] AMOROS C., WADE P.M. - Successions écologiques. In Hydrosystèmes fluviaux. Sous la direction de C. Amoros et G.E. Petts. Masson ed. Paris. 201-231 (1993).

[11] STANKOVIC S., JANKOVIC D. - Mechanismus der Fischproduktion im Gebiet des mittleren Donaulaufe. Arch. Hydrobiol. (Suppl. Donauforschung) suppl. 36, 299-305. (1971).

[12] LÉGER L. - Economie biologique et productivité de nos rivières à Cyprinidés. Bulletin Français de Pisciculture. 139. 1-21 (1948).

[13] GORMAN O.T., KARR J.R. - Habitat structure and stream fish communities. Ecology , 59, 507-515 (1978).

[14] MAIRE G., CLOOTS-HIRSCH A.R. - Etude morphodynamique de la vallée et du lit de la Loire Bourguignonne. In Vallées de la Loire et de l'Allier en Bourgogne. Direction Régionale de I'Equipement de Bourgogne. 114 p. plus cartes. (1983)
[15] SOGREAH - Contribution à l'étude de l'évolution des lits. hydrologie. In Vallées de la Loire et de l'Allier en Bourgogne. Direction Régionale de l'Equipement de Bourgogne. 90 p. plus cartes. (1983).

[16] MALAVOI J.R. - Etude géomorphologique de la Loire entre Villerest et Nevers. Note d'avancement de l'étude. DIREN de Bassin Loire-Bretagne. (1996).

[17] GREMINAT, BUGNON F., PAGES J., CLAVIER J.L., LOISEAU J.E., DUCERF G. - Etude et cartographie des biocénoses dans la vallée de la Loire nivernaise. In Vallées de la Loire et de l'Allier en Bourgogne. Direction Régionale de l'Equipement de Bourgogne. 114 p. plus cartes. (1983).

[18] BABONAUX Y. - Le lit de la Loire. Etude d'hydrodynamique fluviale. Comité des Travaux Historiques et Scientifiques. Paris. Imp. Nationale. 252 p. (1970).

[19] SOUCHON Y., MOUTHON J., COSTE M., FAESSEL B. Etude hydrobiologique et piscicole de la Loire amont. Synthèse de l'état de référence du site de Villerest. Rapport Cemagref, DQEPP/ Institution Interdépartementale pour la protection des vals de Loire contre les inondations. 53 p. (1984).

[20] BOISNEAU P., MENNESSON-BOISNEAU C., BAGLINIÈRE J.L. - Description d'une frayère et comportement de reproduction de la grande Alose (Alosa alosa L.) dans le cours supérieur de la Loire. Bull. Fr. Pêche Piscic. 316. 15-23. (1990).

[21] ALLARDI J. - Ambloplites rupestris (Rafinesque.1817) (Poisson Centrarchidae). Espèce nouvelle pour la faune de France. Cybium. 53-57 (1978).

[22] ROCHÉ J., HEINTZ C. - Les poissons de Loire. Patrimoine naturel de Bourgogne, 2, 22-25. (1994).

[23] ASSISES NATIONALES DE L'EAU (ouvrage collectif)- Protection des milieux naturels aquatiques. Rapport du groupe de travail. Ministère de l'Environnement et de la Prévention des Risques Technologiques et Naturels Majeurs. (1990).

[24] BRAVARD J.P. - Basse vallée de l'Ain : Etude de définition d’un schéma global d’aménagement. Conseil Général de r'Ain. (1991).

[25] FEL.ZINES J.C. - Les végétations du bord de Loire. Patrimoine naturel de Bourgogne,2, 12-15. (1994).

[26] LOIRE NATURE - Un espace de liberté pour la Loire et l'Allier. (1995). 